\title{
Mechanisms underlying pro-arrhythmic abnormalities arising from Pitx2-induced electrical remodelling: an in silico intersubject variability study
}

\author{
Yijie Zhu' ${ }^{1}$, Jieyun $\mathrm{Bai}^{1} \wedge$, Andy $\mathrm{Lo}^{2}$, Yaosheng Lu${ }^{1}$, Jichao Zhao ${ }^{2}$ \\ ${ }^{1}$ Department of Electronic Engineering, College of Information Science and Technology, Jinan University, Guangzhou, China; ${ }^{2}$ Auckland \\ Bioengineering Institute, University of Auckland, Auckland, New Zealand \\ Contributions: (I) Conception and design: Y Zhu, J Bai, J Zhao; (II) Administrative support: J Bai, Y Lu; (III) Provision of study materials or patients: \\ J Bai; (IV) Collection and assembly of data: Y Zhu, J Bai; (V) Data analysis and interpretation: Y Zhu, J Bai; (VI) Manuscript writing, reviewing and \\ revising: All authors; (VII) Final approval of manuscript: All authors. \\ Correspondence to: Jieyun Bai, PhD; Yaosheng Lu, PhD. Department of Electronic Engineering, College of Information Science and Technology, \\ Jinan University, 601 Huangpu Avenue West, Guangzhou, China. Email: bai_jieyun@126.com; tluys@jnu.edu.cn.
}

Background: Electrical remodelling as a result of the homeodomain transcription factor 2 (Pitx2)-
dependent gene regulation induces atrial fibrillation (AF) with different mechanisms. The purpose of this
study was to identify Pitx2-induced changes in ionic currents that cause action potential (AP) shortening and
lead to triggered activity.

Methods: Populations of computational atrial AP models were developed based on AP recordings from sinus rhythm (SR) and AF patients. Models in the AF population were divided into triggered and untriggered AP groups to evaluate the relationship between each ion current regulated by Pitx 2 and triggered APs. Untriggered AP models were then divided into shortened and unshortened AP groups to determine which Pitx2-dependent ion currents contribute to AP shortening.

Results: According to the physiological range of AP biomarkers measured experimentally, populations of 2,885 SR and 4,781 AF models out of the initial pool of 30,000 models were selected. Models in the AF population predicted AP shortening and triggered activity observed in experiments in Pitx2-induced remodelling conditions. The AF models included 925 triggered AP models, 1,412 shortened AP models and 2,444 unshortened AP models. Intersubject variability in $I_{K s}$ and $I_{C a L}$ primarily modulated variability in AP duration (APD) in all shortened and unshortened AP models, whereas intersubject variability in $I_{K 1}$ and SERCA mainly contributed to the variability in AP morphology in all triggered and untriggered AP models. The incidence of shortened AP was positively correlated with $I_{K s}$ and $I_{K l}$ and was negatively correlated with $I_{N a}, I_{C a L}$ and SERCA, whereas the incidence of triggered AP was negatively correlated with $I_{K s}$ and $I_{K l}$ and was positively correlated with $I_{N a}, I_{C a L}$ and SERCA.

Conclusions: Electrical remodelling due to Pitx2 upregulation may increase the incidence of shortened AP, whereas electrical remodelling arising from Pitx2 downregulation may favor to the genesis of triggered AP.

Keywords: Electrical remodelling; atrial fibrillation (AF); Pitx2; triggered activity; mathematical modelling

Submitted Aug 03, 2020. Accepted for publication Oct 30, 2020.

doi: $10.21037 / \mathrm{atm}-20-5660$

View this article at: http://dx.doi.org/10.21037/atm-20-5660

^ ORCID: 0000-0002-2847-350X. 


\section{Introduction}

Genome-wide association studies have identified genetic variants associated with atrial fibrillation $(\mathrm{AF})$ populations, with the strongest hits clustering on chromosome $4 \mathrm{q} 25$, close to the gene for the homeodomain transcription factor 2 (Pitx2) (1). Both under- and overexpression of Pitx2 mRNA have been found to be associated with $\mathrm{AF}(2,3)$. In addition, the study of Syeda et al. (4) has demonstrated a Pitx2 mRNA gradient in AF patients requiring AF ablation. Hypertensive rats also have shown an agedependent decrease of left atrial Pitx2 expressions and the age-dependency of spontaneous atrial tachyarrhythmias, indicating an association between left atrial Pitx2 downregulation and increased atrial arrhythmogenicity (5). Therefore, mRNA expression levels of Pitx2 might provide information on variability in $\mathrm{AF}$ risk.

Linking mRNA changes of membrane effector genes regulated by Pitx 2 to action potential (AP) alterations might favor the understanding of mechanisms of $\mathrm{AF}$ promotion due to atrial cellular electrophysiological remodelling arising from the variability of Pitx2 (6). Experimental studies have shown that Pitx2 regulated several ion channels (including sodium channel gene Scn5a, calcium channel gene Cacna1c, and potassium channel genes Kcnq1 and Kcnj2) and calcium handling genes (including Ryr2 and Atp2a2) in mutant Pitx2 models $(3,4,7,8)$. Although AP duration (APD) shortening (9), depolarized resting membrane potential (RMP) (4) and triggered activity $(8,10)$ were observed, the ionic and cellular mechanisms may be different because mRNA expression levels might vary with circadian rhythm (11,12), gender differences (13-15) and spatial location within the heart (16).

Both experimental and theoretical studies of the mechanisms underlying Pitx2-induced AF often ignored intersubject variability in atrial electrophysiology properties (2-4,7-10,17-21). Recently, experimentally reported variability was incorporated into mathematical models of biological systems to develop a population of models for investigating cellular electrophysiology $(22,23)$. This approach aimed to provide a new framework to link molecular mechanisms to cellular-level outputs by revealing unique subsets of model parameters linked to known phenotypes of human atrial myocytes (23-28). Simulation studies using populations of models allow exploring potential causes and implications of the cell level variability exhibited in experimental recordings $(23,29)$.

Thus, the equations proposed in the recent human atrial cellular kinetics model (TPA) (20) were considered as the model structure to generate over 30,000 candidate models, all sharing the same equations as in the original TPA model, but with different parameter values for the ionic properties associated with Pitx2 regulation, randomly selected within a wide range. The TPA model population was then calibrated using a set of cellular biomarkers extracted from experimental AP recordings [from sinus rhythm (SR) and AF patients] (23) to capture key AP properties. The experimentally calibrated human atrial $\mathrm{AP}$ models in the $\mathrm{AF}$ population were divided into triggered and untriggered $\mathrm{AP}$ groups which then divided into shortened and unshortened AP groups. Differences between triggered and untriggered AP models were used to quantify the contribution of specific ionic currents to determining intersubject variability in cellular human atrial AP morphology. The shortened and unshortened AP models were then used to identify the ionic mechanisms determining intersubject variability in human atrial APD. Here, we specifically showed that the calibrated cell model population quantitatively predicts triggered activity and AP shortening caused by Pitx 2 -induced electrical remodelling. The tools may be applied for in silico screening and prediction of drug effects in Pitx2-induced AF patients.

We present the following article in accordance with the MDAR reporting checklist (available at http://dx.doi. org/10.21037/atm-20-5660).

\section{Methods}

\section{Mathematical models of the human atrial cell and Pitx2- induced remodelling}

The TPA model developed by our group was chosen as a base to construct the computational AP model populations as it is able to reproduce human AP morphology, APD rate dependence and triggered activity, i.e., early afterdepolarizations (EADs), delayed afterdepolarizations (DADs) and spontaneous depolarizations (20). This biophysically-detailed model of human atrial cellular electrophysiology also has been used to investigate mechanisms underlying Pitx2-induced AF (17-20,30,31) and here we provide a brief description. It includes representation of the 13 transmembrane ionic currents and two main intracellular calcium flows, including fast sodium current $\left(I_{N_{a}}\right)$, transient outward potassium current $\left(I_{t o}\right)$, rapid delayed rectifier potassium current $\left(I_{K r}\right)$, slow delayed rectifier potassium current $\left(I_{K s}\right)$, ultrarapid delayed 
rectifier potassium current $\left(I_{K u r}\right)$, inward rectifier potassium current $\left(I_{K 1}\right)$, L-type calcium current $\left(I_{C a L}\right)$, background sodium current $\left(I_{N a b}\right)$, background calcium current $\left(I_{C a b}\right)$, plateau potassium current $\left(I_{p K}\right)$, plateau calcium current $\left(I_{p C a}\right)$, sodium-potassium pump current $\left(I_{N a K}\right)$, sodium-calcium exchange current $\left(I_{N C X}\right)$, the calcium flow $\left(\mathcal{F}_{u p}\right)$ through the sarcoplasmic reticulum calcium ATPase (SERCA) and calcium release flow $\left(\mathcal{F}_{\text {rel }}\right)$. mRNA expression data $(3,4,7,8,10)$ are used to determine directions of changes

Table 1 Parameters associated with the homeodomain transcription factor 2 (Pitx2)-dependent gene regulatory network $(3,4,7,8)$

\begin{tabular}{lccc}
\hline Gene & Current & Parameter & $\begin{array}{c}\text { Regulation in Pitx2- } \\
\text { induced AF }\end{array}$ \\
\hline Scn5a & $\mathrm{I}_{\mathrm{Na}}$ & $\mathrm{G}_{\mathrm{Na}}$ & $\uparrow$ \\
Cacna1c & $\mathrm{I}_{\mathrm{CaL}}$ & $\mathrm{G}_{\mathrm{CaL}}$ & $\downarrow$ \\
Kcnq1 & $\mathrm{I}_{\mathrm{Ks}}$ & $\mathrm{G}_{\mathrm{Ks}}$ & $\uparrow$ \\
Kcnj2 & $\mathrm{I}_{\mathrm{K} 1}$ & $\mathrm{G}_{\mathrm{K} 1}$ & $\downarrow$ \\
Ryr2 & RyR2 $\left(\mathrm{J}_{\mathrm{rel}}\right)$ & $\mathrm{G}_{\mathrm{rel}}$ & $\uparrow$ \\
Atp2a2 & SERCA2 $\left(\mathrm{J}_{\mathrm{up}}\right)$ & $\mathrm{G}_{\mathrm{up}}$ & $\uparrow$ \\
\hline
\end{tabular}

$I_{\mathrm{Na}}$, fast sodium current; $I_{\mathrm{Ks}}$, slow delayed rectifier potassium current; $I_{K 1}$, inward rectifier potassium current; $I_{\text {CaL }}$, L-type calcium current; $J_{\text {up }}$, calcium flow through the sarcoplasmic reticulum calcium ATPase (SERCA); $J_{\text {rel }}$, calcium release flow through ryanodine receptors (RyR); $G_{N a}$, maximum conductance of $I_{N a} ; G_{K 1}$, maximum conductance of $I_{\mathrm{K} 1} ; \mathrm{G}_{\mathrm{Ks}}$, Maximum conductance of $\mathrm{I}_{\mathrm{Ks}}$; $\mathrm{G}_{\text {CaL }}$, maximum conductance of $\mathrm{I}_{\mathrm{CaL}} ; \mathrm{G}_{\mathrm{rel}}$, maximum conductance of $\mathrm{J}_{\text {rel }} ; \mathrm{G}_{\mathrm{up}}$, maximum conductance of $\mathrm{J}_{\mathrm{up}}$.

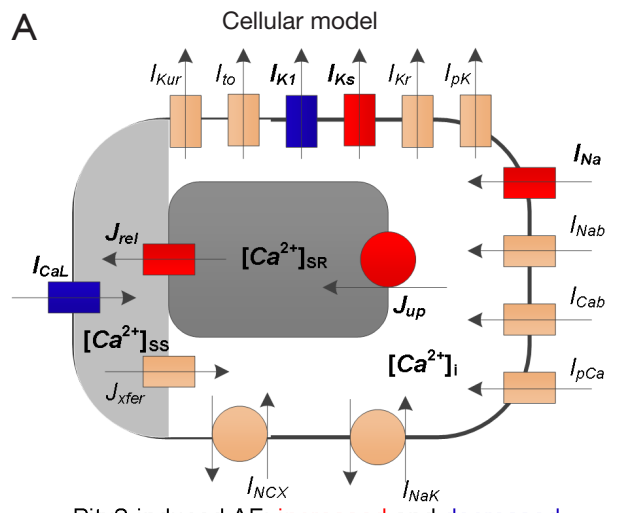

Pitx2-induced AF: increased and decreased currents sampled for population of models in ionic conductances in SR versus Pitx2-induced AF cardiomyocytes, as summarized in Table 1. Pitx2-induced electrical remodelling included an increase in $I_{N a}, I_{K s}, \mathcal{F}_{r e l}$ and $\mathcal{f}_{u p}$, and a reduction in $I_{K 1}$ and $I_{C a L}$ (Figure $1 A$ ).

\section{Experimental data and biomarkers}

For our experimental dataset, AP recordings from $n=254$ right atrial appendages of $\mathrm{N}=214 \mathrm{SR}$ patients and from $\mathrm{n}=215$ right atrial appendages of $\mathrm{N}=149$ AF patients, were published in the previous study (23). In the previous experimental study, right atrial appendages were obtained when patients were undergoing cardiac surgery for coronary artery bypass grafting or mitral/aortic valve replacement. Antiarrhythmic drugs were discontinued before the study. Human myocytes were isolated enzymatically from atrial appendages as previously described (32) and APs were recorded with standard intracellular microelectrodes in atrial trabeculae $(33,34)$. Bath solution contained (in $\mathrm{mM}$ ): $\mathrm{NaCl} 127, \mathrm{KCl} 4.5, \mathrm{MgCl}_{2} 1.5, \mathrm{CaCl}_{2}$ 1.8, glucose 10, $\mathrm{NaHCO}_{3} 22, \mathrm{NaH}_{2} \mathrm{PO}_{4} 0.42$, equilibrated with $\mathrm{O}_{2}-\mathrm{CO}_{2}$ [95:5] at $36.5 \pm 0.58{ }^{\circ} \mathrm{C}, \mathrm{pH}$ 7.4. Preparations were regularly stimulated at $1 \mathrm{~Hz}$ for at least $1 \mathrm{~h}$ before data acquisition $(33,34)$. The following parameters were quantified to characterize intersubject variability in human atrial AP: action potential amplitude (APA), maximum upstroke velocity $\left(\mathrm{dVdt}_{\max }\right), \mathrm{RMP}$, and APD at $20 \%\left(\mathrm{APD}_{20}\right), 50 \%$ $\left(\mathrm{APD}_{50}\right)$ and $90 \%\left(\mathrm{APD}_{90}\right)($ Figure $1 B)$. Minimum, maximum and mean \pm standard deviation values for these biomarkers

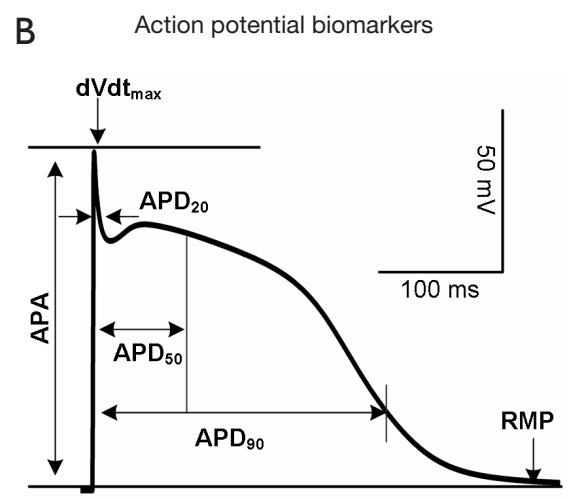

Figure 1 Parameters related to Pitx2-induced electrical remodelling and biomarkers of the action potential. (A) Modifications to the Bai model for simulating atrial fibrillation due to Pitx2-induced electrical remodelling are depicted in red and blue circles (18,20); (B) action potential biomarkers: action potential amplitude (APA), maximum upstroke velocity $\left(\mathrm{dVdt}_{\max }\right)$, resting membrane potential (RMP), and action potential duration at $20 \%\left(\mathrm{APD}_{20}\right), 50 \%\left(\mathrm{APD}_{50}\right)$ and $90 \%\left(\mathrm{APD}_{90}\right)$. 


\section{Page 4 of 17}

Table 2 Minimum and maximum values of action potential biomarkers in sinus rhythm (SR) versus atrial fibrillation (AF) patients (23)

\begin{tabular}{lcc}
\hline Biomarkers & SR & AF \\
\hline APA & 75 to $120(95 \pm 7)$ & 80 to $130(102 \pm 8)$ \\
dVdt $_{\text {max }}$ & 40 to $420(220 \pm 68)$ & 40 to $420(232 \pm 70)$ \\
RMP & -85 to $-65(-74 \pm 4)$ & -85 to $-65(-77 \pm 4)$ \\
APD $_{20}$ & 1 to $60(7 \pm 8)$ & 1 to $75(30 \pm 18)$ \\
APD $_{50}$ & 6 to $200(139 \pm 44)$ & 30 to $200(102 \pm 28)$ \\
APD $_{90}$ & 190 to $440(318 \pm 42)$ & 140 to $330(216 \pm 35)$ \\
\hline
\end{tabular}

Values are ranges with means $\pm S D$ in biomarkers. APA, action potential amplitude; $\mathrm{dVdt}_{\max }$, maximum upstroke velocity; RMP, resting membrane potential; $A \mathrm{PD}_{20}, \mathrm{APD}_{50}$ and $A P D_{90}$, action potential duration at $20 \%, 50 \%$ and $90 \%$, respectively.

are presented in Table 2. More information (including ethics approval, informed consent and basic information of participants) regarding the experimental conditions under which the data were collected is available in the study of Sánchez et al. (23).

\section{Construction of populations of models of human atrial electrophysiology}

To capture intersubject variability, populations of sampled models of human atrial electrophysiology for both SR and AF were generated based on the original TPA model. All models in each population shared the same equations but parameters of ionic current conductances in determining the human atrial AP were varied with respect to their original values. These parameters were sampled over a range from $-100 \%$ to $+200 \%$ of their original values with the Latin hypercube sampling (LHS) methodology (22) to create 30,000 candidate models that were sufficient for convergence of the sensitivity coefficients for both SR and AF model populations (22). Following the ASME V\&V40 Standard proposed by the Subcommittee of the American Society of Mechanical Engineers (ASME) on Verification and Validation $(V \& V)$ in Computational Modeling of Medical Devices (35) for developing a structured approach for establishing the credibility of computational models for a specific use, we used the candidate models to simulate human atrial AP by considering stimulation frequency $(1 \mathrm{~Hz})$ under the experimental conditions (23) and calculate AP biomarkers of each candidate model. The candidate models generated in the previous step were selected

\section{Zhu et al. Mechanisms underlying Pitx2-induced atrial fibrillation}

to constitute SR and AF populations whose simulated electrophysiological properties are in range with the same properties in experimental data on AP biomarkers (including APA, $\mathrm{dVdt}_{\max }, \mathrm{RMP}, \mathrm{APD}_{20}, \mathrm{APD}_{50}$ and $\mathrm{APD}_{90}$ ) in Table 2. This step yields the experimentally-calibrated population of models. The impact of intersubject variability in the 6 Pitx2-induced remodelled targets (2-5,7-10,21) was evaluated using these models in the $\mathrm{AF}$ population. These models firstly were divided into triggered and untriggered $\mathrm{AP}$ groups to identify the main ion channel conductances involved in the genesis of triggered activity. Triggered patterns were identified when a positive derivative of the membrane potential (36) or a deflection of the membrane potential larger than $1 \mathrm{mV}$ during the diastolic phase (37) was observed. Then, the models in the untriggered AP group were divided into shortened and unshortened AP groups to examine the contribution of each remodelled target to the APD shortening (Figure 2). Shortened patterns were identified when $\mathrm{APD}_{90}$ of the untriggered $\mathrm{AP}$ model in the AF population is smaller than that of the AP model in the SR population. In detail, shortened AP patterns were identified when their $\mathrm{APD}_{90}$ is within the range (140-330 ms) of AF data but not within the range (190-440 ms) of SR (Table 2).

\section{Stimulation protocols}

The stimulation protocol mimics the one used by Sánchez et al. (23) to obtain AP measurements in SR and AF cardiomyocytes. Cell mathematical models were initially preconditioned by pacing at a basic cycle length of 1,000 ms until the steady-state was reached. A stimulus with an amplitude of $-80 \mu \mathrm{A} / \mathrm{cm}^{2}$ and a duration of $0.5 \mathrm{~ms}$ is applied at each pace.

\section{Statistical analysis, software, numerical methods and code availability}

To quantify the relative importance of ionic conductances in determining changes in the biomarkers, partial correlation coefficients (PCCs) were used on the SR and AF populations to evaluate the role of each ionic current on properties of the whole AP (22). Partial correlation is a method to find correlations between two variables, after accounting for the linear effects of one or more additional variables (38). PCC between $x$ and $y$, given the set of $N$ additional variables $z_{i}$, is then defined as the correlation coefficient between the residuals $r_{x}=x-\hat{x}$ and $r_{y}=y-\hat{y}(22) . \hat{x}$ and $\hat{y}$ are the 


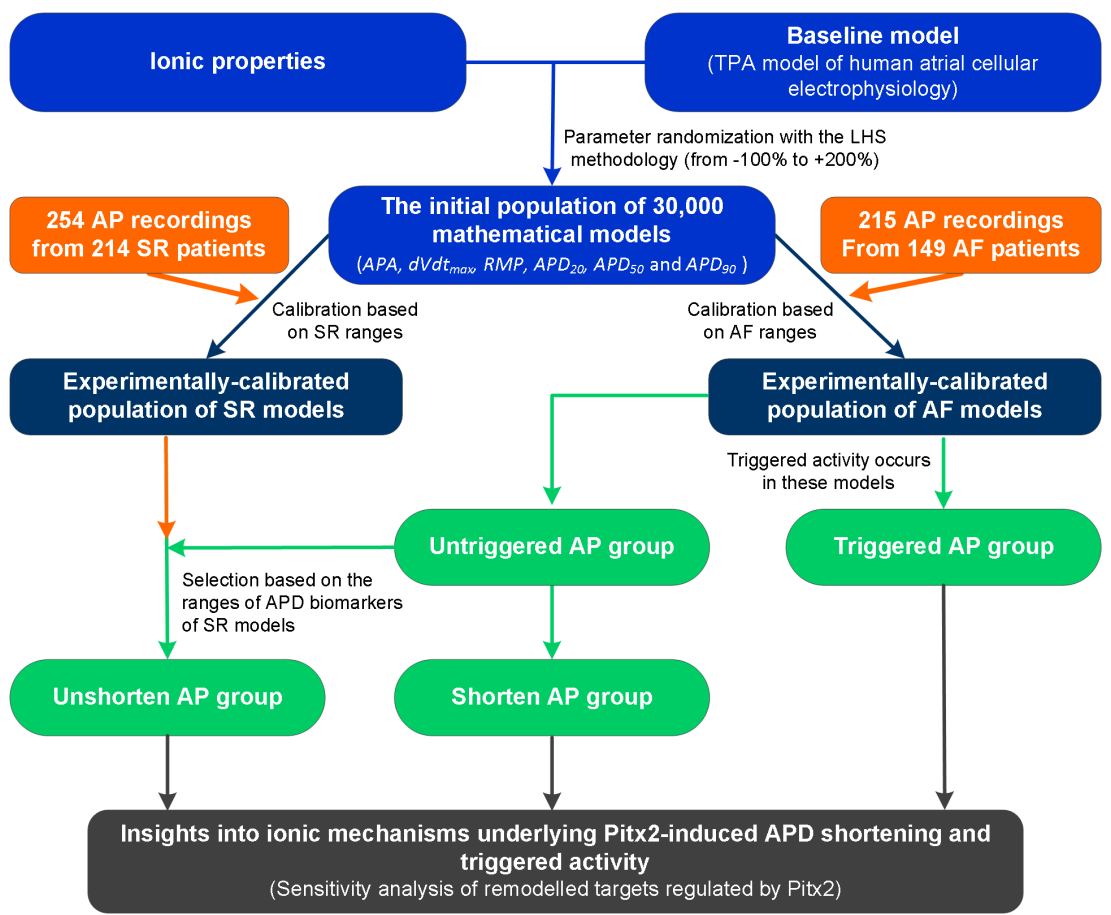

Figure 2 Flow chart illustrating the process behind investigating the proarrhythmic effects of Pitx2-induced electrical remodelling. TPA model: a recent human atrial cellular kinetics model developed by our group (20); AP, action potential; APD, AP duration; SR, Sinus rhythm; AF, atrial fibrillation; Pitx2, the homeodomain transcription factor 2 .

respective sample means or the following linear regression models:

$$
\begin{aligned}
& \hat{x}=c_{0}+\sum_{i=1}^{N} c_{i} z_{i} \text { and } y=b_{0}+\sum_{i=1}^{N} b_{i} z_{i} \\
& \operatorname{PPC}\left(x, y, z_{i}\right)=\frac{\operatorname{Cov}\left(r_{x}, r_{y}\right)}{\operatorname{Var}\left(r_{x}\right) \operatorname{Var}\left(r_{y}\right)}
\end{aligned}
$$

where $\operatorname{Cov}\left(r_{x}, r_{y}\right)$ represents the covariance between $r_{x}$ and $r_{y}$, while $\operatorname{Var}\left(r_{x}\right)$ and $\operatorname{Var}\left(r_{y}\right)$ are respectively the variance of $r_{x}$ and variance of $r_{y}$.

To evaluate Pitx2-induced remodelled targets influencing arrhythmia proclivity, listic regression was applied to study the mechanisms responsible for the development of tggered activity and AP shortening (39). Statistical significance in differences on ionic conductance distributions between populations was evaluated by using the Mann-Whitney $\mathrm{U}$ test. A probability $<0.05$ was considered statistically significant.

Previous studies have shown that changes in Pitx2 levels lead to electrical remodelling linked to arrhythmogenesis (2) and that Pitx2 regulates membrane effector genes associated with the electrical remodelling in a dose-dependent manner
$(3,7,8)$. Therefore, we assumed that changes in the Pitx2 level are translated to the extent of electrical remodelling (including $G_{N a}, G_{K s}, G_{K l}, G_{C a L}, G_{r e l}$ and $\left.G_{u p}\right)(3,7,8)$. The correlation of the Pitx2 levels with the different AF models developed based on Pitx2-induced electrical remodelling was built by evaluating the contribution of remodelled targets (including $G_{N a}, G_{K s}, G_{K l}, G_{C a L}, G_{r e l}$ and $G_{u p}$ ) to the changes in APs (including unshortened AP, shortened AP and triggered $\mathrm{AP})$. The extent of electrical remodelling was quantified with weights of remodelled targets for classification of different AF subgroups (unshortened, shortened and triggered groups). Here, we used the AF models (as output) and the corresponding remodelled parameters (as input) to train the artificial neural network (40) to determine the weights of remodelled parameters. Based on the correlation of the Pitx2 levels with remodelled parameters observed in previous studies $(3,4,7,8,41)$ and the correlation of remodelled parameters with changes in APs, the correlation of the Pitx2 levels with different $\mathrm{AF}$ models was built.

All simulations and data analyses were performed on a computing cluster with Intel(R) Xeon(R) CPU E5-2690 v4 @ 2.60GHz 32 CPUs 28 CPUs (56 threads) + 128GB. The 

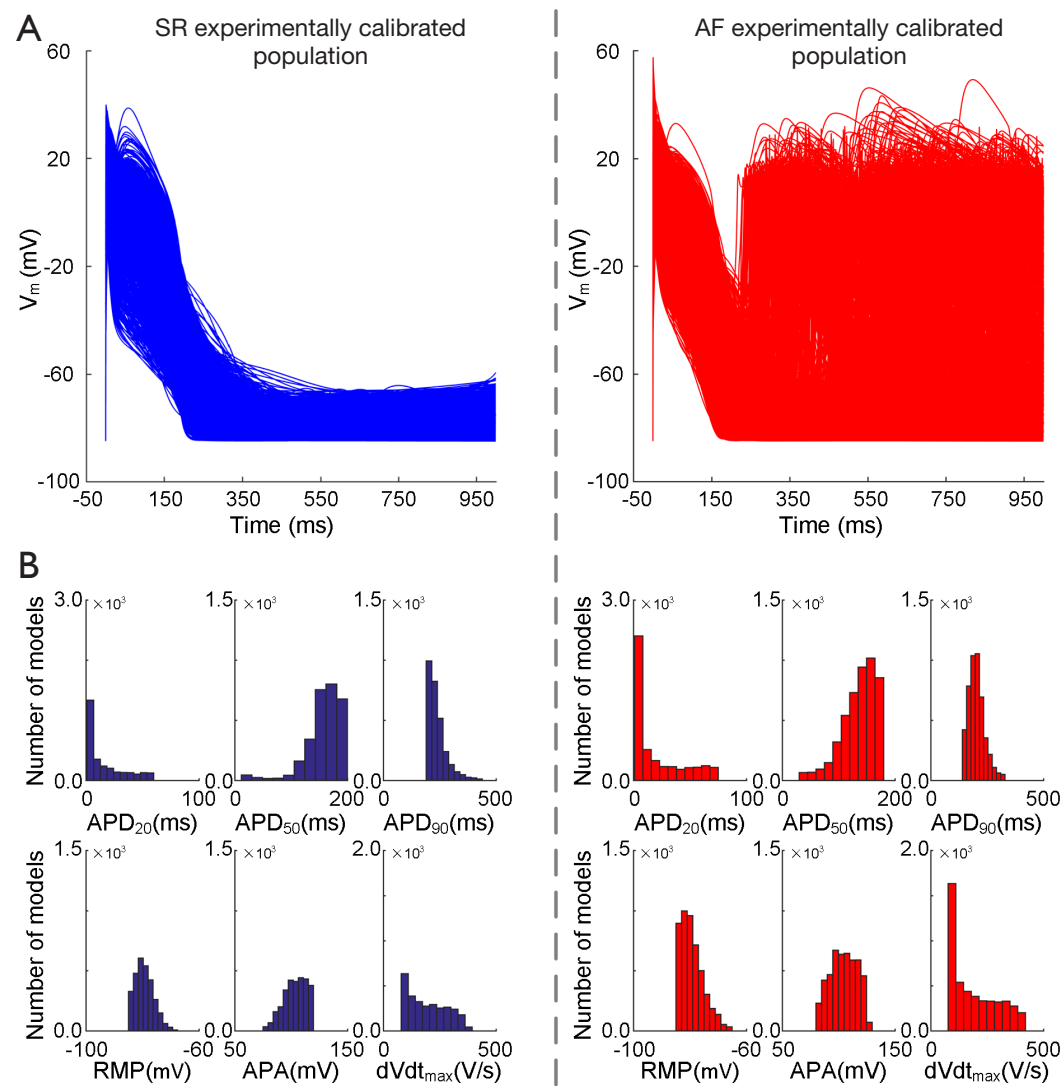

Figure 3 The experimentally calibrated populations of human atrial cell models for sinus rhythm (SR) and atrial fibrillation (AF). (A) Representative traces of action potential (AP) models; (B) distributions of AP biomarkers (including $\mathrm{APA} \mathrm{dVdt}_{\max }, \mathrm{RMP}^{\mathrm{APD}} \mathrm{AP}_{20}, \mathrm{APD}_{50}$ and $\left.\mathrm{APD}_{90}\right)$.

mathematical model (available from the repository CellML http://models.cellml.org/workspace/520) was implemented in MATLAB 2018a (The MathWorks, Natick, MA, USA) using the stiff ordinary differential equation solver ode15s and analysis of biomarkers was also performed using MATLAB. Our user project containing newly created datasets and the simulation codes used in this study is available to download from the GitHub website (https:// github.com/aspirerabbit).

\section{Results}

\section{The main hallmarks of the human Pitx2-induced AF electrophysiological phenotype}

As a result of the experimentally driven calibration, the human atrial SR and AF populations respectively consist of 2,885 and 4,781 models, qualitatively and quantitatively in agreement with the experimental recordings, accounting for biological variability. Figure $3 A$ shows representative $\mathrm{AP}$ traces obtained in simulations using the restricted range of variability obtained with the experimentally calibrated $\mathrm{SR}$ and AF populations (Table 2). This AF population reproduced the $\mathrm{AF}$ electrophysiological phenotypes, as shown by the AF biomarkers comparison in Figure $3 B$. In agreement with the experiments $(4,8,9)$ and simulations $(17-$ 20,30,31), AF models are characterized by shortened AP $\left(\mathrm{APD}_{50}\right.$ and $\left.\mathrm{APD}_{90}\right)$, depolarized RMP, increased upstroke velocity $\left(\mathrm{dVdt}_{\max }\right)$, slightly increased APA and triggered activity. Within the AF population, approximately $19 \%$ of the models exhibit repolarization abnormalities, AP biomarkers of these models are not considered for the biomarkers comparison presented above, and are analyzed in detail below.

To investigate ionic properties of human atrial cells contribute to changes in AP biomarkers, PPCs were used to quantify correlations between the parameter 
values associated with Pitx2-induced remodelling and AP biomarkers of each model in the populations, as shown in Figure $4 A, B$. Ionic mechanisms underlying changes in AP biomarkers are similar in the SR population versus the AF population. $\mathrm{APD}_{50}, \mathrm{RMP}$ and $\mathrm{dVdt}_{\max }$ are correlated and are primarily determined by $I_{C a L}, I_{K l}$ and $I_{N a}$. Compared with the SR population, the AP properties in the Pitx2-induced AF population are primarily favored by upregulated $I_{K s}$ and $\mathcal{F}_{u p}$, and downregulated $I_{K 1}(\mathrm{P}<0.001)$ (Figure $4 C$ ).

\section{Role of Pitx2-induced remodelled currents on triggered activity mechanisms}

Models in the AF population (including 4,781 models) were divided into triggered and untriggered AP groups. Representative in silico AP traces from triggered (925 models) and untriggered (3,856 models) AP subgroups are presented in Figure $5 A, B$. In order to identify the main ion channel conductivities involved in the genesis of triggered activity, the 6 modified conductances associated with Pitx2-induced electrical remodelling were compared between the groups of models exhibited triggered versus untriggered APs. Regression coefficients were used to quantify correlations between the parameter values associated with Pitx2-induced remodelling and the genesis of triggered APs, as shown in Figure 5C. The inducibility of triggered APs is negatively correlated with potassium conductances (including $I_{K I}$ and $I_{K s}$ ) and is positively correlated with calcium conductances (including $I_{C a L}$ and $\left.\mathcal{f}_{u p}\right)$, respectively. Although these five parameters $\left(I_{N a}, I_{K 1}, I_{K}\right.$, $I_{C a L}$ and $\left.\mathcal{f}_{u p}\right)$ present significant differences between groups $(\mathrm{P}<0.001)$ (Figure $5 D)$, only directions of changes in $I_{N a}, I_{K 1}$ and $\mathcal{f}_{u p}$ are consistent with the Pitx2-dependent regulation of these currents, as summarized in Table 1 .

To further explore the two main Pitx2-induced remodeled cellular components $\left(I_{K 1}\right.$ and $\left.\mathcal{F}_{u p}\right)$ that contribute to triggered activity, additional simulations were performed artificially impeding downregulated $I_{K I}$ and (or) upregulated $\mathcal{f}_{u p}$. Under the basal condition (Black lines), when including either $I_{K 1}$ down-regulation, $\mathcal{f}_{u p}$ up-regulation, or a combination of both, the depolarized RMP increases much faster during the resting phase, and spontaneous depolarizations are observed, respectively (Figure 6A,B,C,D). When RMP was reduced by increasing $I_{K 1}$ (Red lines), spontaneous depolarizations in the scenario including $I_{K l}$ down-regulation are suppressed (Figure $6 B$ ), whereas a DAD is induced in the scenario including $\mathcal{F}_{u p}$ up-regulation (Figure $6 C$ ). Therefore, the genesis of spontaneous depolarizations is primarily favored by low $I_{K 1}$, and secondarily by high $\mathcal{f}_{u p}$. High $f_{u p}$ also contributes to the development of DADs.

\section{Role of Pitx2-induced remodelled currents on AP shortening mechanisms}

By considering the physiological range of AP biomarkers of the SR population, the shortened AP models were selected from 3,856 untriggered AP models in the AF population. Representative AP traces from unshortened (2,444 models) and shortened (1,412 models) AP groups show the main differences between the two populations (Figure 7A,B). Regression coefficients were used to quantify correlations between the parameter values associated with Pitx2-induced remodelling and AP shortening, as shown in Figure $7 C$. AP shortening is negatively correlated with $I_{C a L}$ and positively correlated with $I_{K s}$, respectively. $I_{N a}, I_{C a L}, I_{K s}$ and $\mathcal{f}_{r e l}$ present significant differences between groups $(\mathrm{P}<0.001)$ (Figure $7 D$ ), but directions of changes in $I_{C a L}, I_{K s}$ and $\mathcal{f}_{r e l}$ are consistent with the Pitx2-dependent regulation of these currents, as summarized in Table 1.

\section{The correlation of Pitx2-induced remodelling with different AF models}

By using the artificial neural network, we got the weights of six remodelled parameters for classifying $\mathrm{AF}$ models (including shortened and triggered AP two categories) (Figure 8). For the shortened AP category, weights of $G_{K s}$ and $G_{K l}$ are negative and weights of $G_{N a}, G_{C a L}$ and $G_{u p}$ are positive, indicating the incidence of shortened AP is positively correlated with $G_{K s}$ and $G_{K l}$ and is negatively correlated with $G_{N a}, G_{C a L}$ and $G_{u p}$. In contrast, for the triggered AP category, weights of $G_{K s}$ and $G_{K 1}$ are positive and weights of $G_{N a}, G_{C a L}$ and $G_{u p}$ are negative. In addition, weights of $G_{r e}$ for shortened AP and triggered AP categories are positive. In detail, the values of weights of different remodelled targets can be found in Table 3.

\section{Discussion}

\section{Major findings}

In the present study, we have built populations of human atrial cell models to investigate the potential of mRNA expression levels regulated by the Pitx2-dependent network to predict Pitx2-induced electrical remodelling in AP, as measured experimentally $(4,8,9)$. Our population-based 

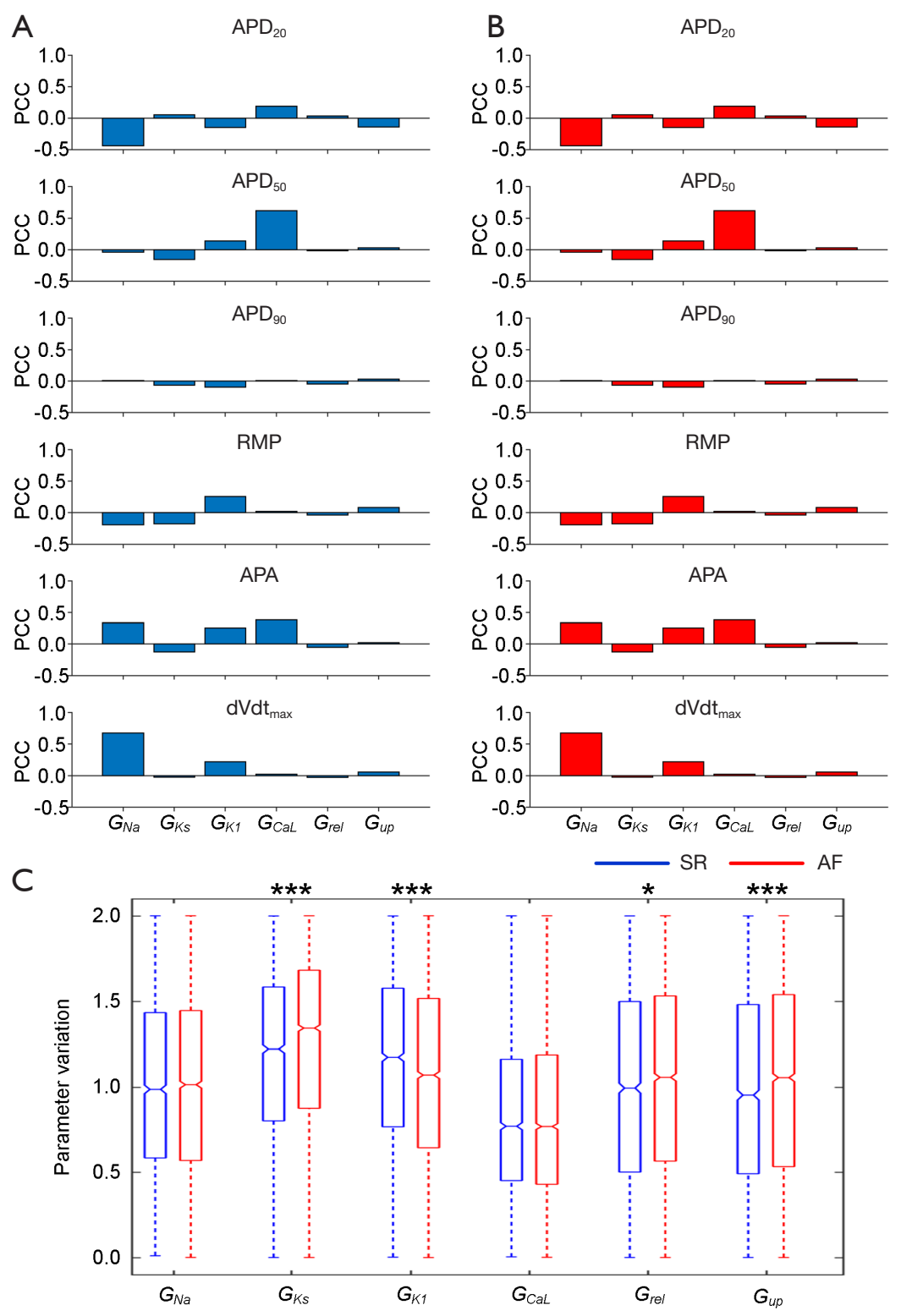

Figure 4 Partial correlation coefficients (PCCs) between action potential biomarkers and current conductance parameters. (A) PPCs between action potential biomarkers (including $\mathrm{APA}, \mathrm{dVdt} t_{\max }, \mathrm{RMP}, \mathrm{APD}_{20}, \mathrm{APD}_{50}$ and $\mathrm{APD}_{90}$ ) and current conductance parameters (including $G_{\mathrm{Na}}, G_{\mathrm{Ks}}, G_{\mathrm{K} 1}, G_{\mathrm{CaL}}, G_{\mathrm{Jrel}}$ and $G_{\mathrm{up}}$ ) for sinus rhythm (SR). (B) PPCs between action potential biomarkers and current conductance parameter for atrial fibrillation (AF). (C) Ranges of the variability of ionic conductances in the human atrial AP model populations. Median values and ranges of the variability of ionic conductances $G_{\mathrm{Na}}, G_{\mathrm{Ks}}, G_{\mathrm{K} 1}, G_{\mathrm{CaL}}, G_{\mathrm{Jrel}}$ and $G_{\mathrm{up}}$ in the experimentally calibrated populations of human atrial AP models, sampled within a $\pm 100 \%$ range of their original values in the TPA human atrial AP models in SR (blue) and AF (red). Each boxplot represents the range covered by the ionic conductances: the edges of the box are the $1^{\text {st }}$ and $3^{\text {rd }}$ quartiles, the whiskers extend to the most extreme datapoints, the estimated median physiological value is the central horizontal line and the notch around the median is the $5 \%$ significance level (Mann-Whitney $\mathrm{U}$ test: *, $\mathrm{P}<0.05$; ***, $\mathrm{P}<0.001)$. 

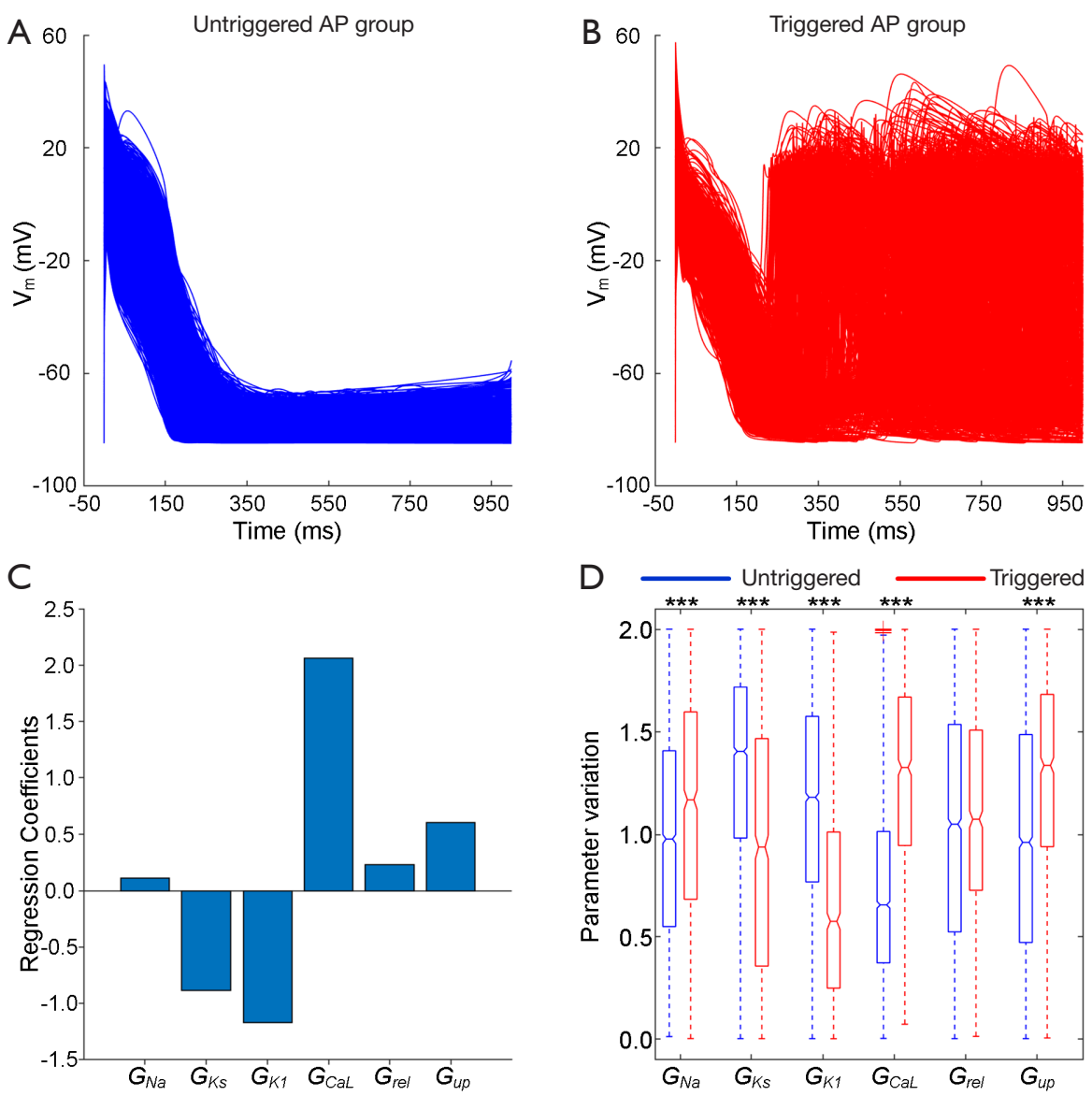

Figure 5 Triggered action potentials in atrial fibrillation and underlying ionic parameters. (A,B) Representative traces of action potential (AP) models in the untriggered versus triggered groups. (C) Coefficients of the multinomial logistic regression model describing the probability of the development of triggered activity in the models of our population. (D) Statistical analysis on the values of the perturbations to parameters modulated by Pitx2 in the two sub-groups of AP models with or without triggered activity. Each boxplot represents the range covered by the ionic conductances: the edges of the box are the $1^{\text {st }}$ and $3^{\text {rd }}$ quartiles, the whiskers extend to the most extreme datapoints, the estimated median physiological value is the central horizontal line and the notch around the median is the 5\% significance level (MannWhitney $\mathrm{U}$ test: $\left.{ }^{* * *}, \mathrm{P}<0.001\right)$.

approach takes into consideration variability arising from the uncertainty associated with the extent to which mRNA expression affects functional channel conductance at the cell membrane. This is in contrast to the traditional approach of considering unique values of ionic conductances in atrial AP models of SR and Pitx2-induced AF human atrial cardiomyocytes (4,17-20,30,31). Our main findings are:

(I) We have built populations of human atrial cell models that reproduce and predict the variability in human atrial AP properties exhibited in a set of AP measurements from over $363 \mathrm{SR}$ and $\mathrm{AF}$ patients. RMP, $\mathrm{dVdt}_{\max }$ and $\mathrm{APD}_{50}$ are correlated in both SR and AF cardiomyocytes, and are primarily determined by $I_{K l}, I_{N a}$ and $I_{C a L}$, respectively.

(II) The Pitx2-dependent mRNA data associated with $I_{N a}, I_{K 1}, I_{K s}, I_{C a L}, \operatorname{RyR}\left(\mathcal{F}_{r e l}\right)$ and SERCA $\left(\mathcal{F}_{u p}\right)$ predict $\mathrm{AP}$ shortening and triggered APs in AF versus SR cardiomyocytes, as reported in experimental studies $(4,8,9)$.

(III) The inducibility of triggered activity is positively correlated with $I_{N a}, I_{C a L}$ and SERCA $\left(\mathcal{F}_{u p}\right)$, and negatively correlated with $I_{K 1}$ and $I_{K}$. Triggered activity due to Pitx2-induced electrical remodelling is primarily favored by high SERCA $\left(\mathcal{F}_{u p}\right)$ and low $I_{K 1}$ in AF myocytes.

(IV) AP shortening is positively correlated with $I_{K s}$ and 

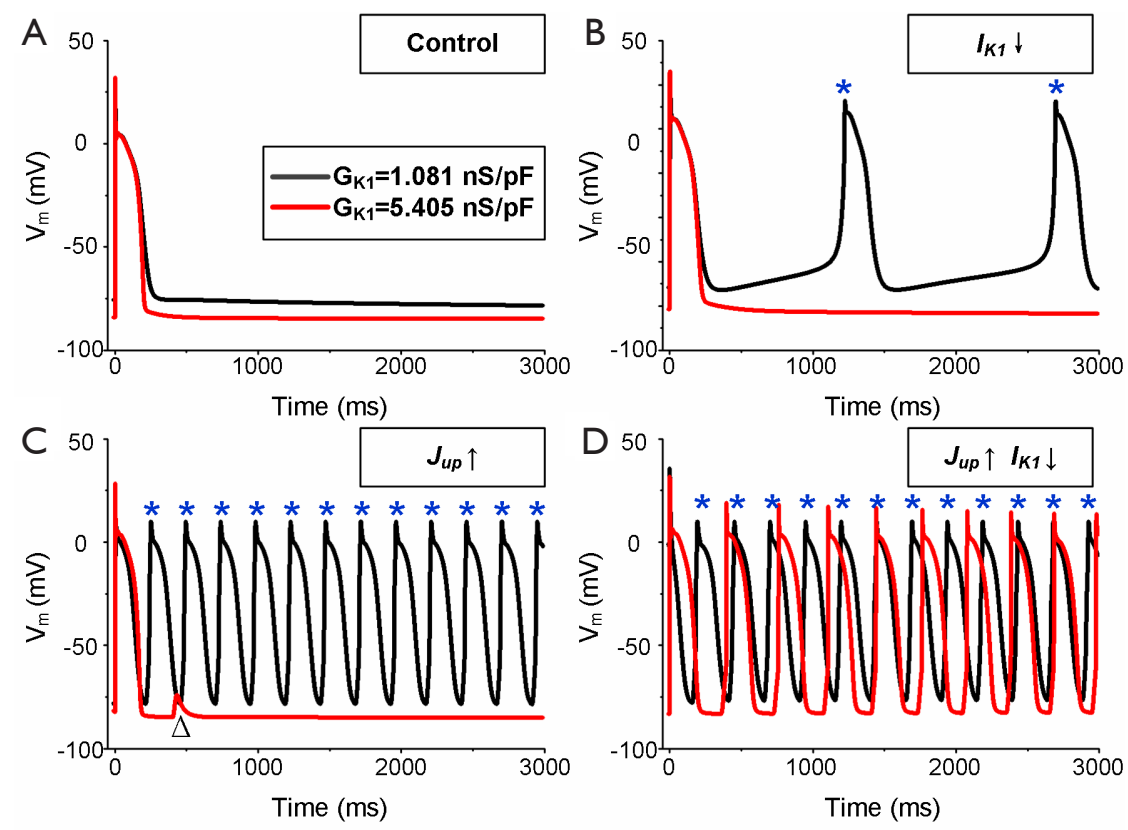

Figure 6 Role of $\mathrm{I}_{\mathrm{K} 1}$ and SERCA $\left(\mathrm{J}_{\mathrm{up}}\right)$ in the genesis of triggered activity. (A) Simulations are shown both with normal $\mathrm{G}_{\mathrm{K} 1}$ (black) and under reduced $G_{K 1}$ conditions (red). (B) APs when $I_{K 1}$ is down-regulated. (C) APs when $J_{\text {up }}$ is up-regulated. (D) APs when $J_{\text {up }}$ is up-regulated and $I_{K 1}$ is down-regulated. Asterisks indicate spontaneous depolarizations and the triangle denotes a DAD. *, spontaneous depolarizations.

$I_{K 1}$, and negatively correlated with $I_{N a}, I_{C a L}$ and SERCA. AP shortening arising from Pitx2-induced electrical remodelling is primarily favored by high $I_{K s}$ and low $I_{C a L}$ in $\mathrm{AF}$ myocytes.

\section{AF population of models}

Mathematical models have been used to evaluate hypotheses of $\mathrm{AF}$ perpetuation mechanisms since the late 1950s. During the last decades, as a result of the increase in computing power together with more extended research in cardiac ionic currents by patch-clamp experiments, more sophisticated electrophysiological models have been used to evaluate the proarrhythmic effects of Pitx2induced remodelling on both AP and rotors dynamics $(4,17-20,30,31)$. However, most of these in silico studies have made use of a single set of parameters fitted to an average of many experiments. Although this approach has allowed the reproduction and better understanding of AF mechanisms, it hides the variability between patients observed in clinical practice. This variability may explain many different phenotypes reported in the literature (23-29). Our Simulations of AF populations of models accounted for the variability observed across subjects. Furthermore, this is the first study in which a population of models has been used to determine the ionic currents linked to arrhythmia perpetuation observed in mutant Pitx 2 models. In the present study, the TPA model was used as the basal model to investigate the mechanisms underlying Pitx2-induced AF. It had been validated and fully assessed by comparing with existing human atrial models [including Lugo et al. model (42), Maleckar et al. model (43), Nygren et al. model (44), Koivumäki et al. model (45), Courtemanche et al. model (46), Grandi et al. model (47), CRN_TP model $(18,20)$ and Voigt et al. model $(48)]$. This model was chosen as it is able to reproduce human AP morphology, APD rate dependence and triggered activity, i.e., early afterdepolarizations (EADs), delayed afterdepolarizations (DADs) and spontaneous depolarizations. It also was used to investigate the pro-arrhythmic effects of Pitx2-induced remodelling and it can reproduce phenomenons (including triggered activity and AP shortening) observed in the experiments. In our previous study, simulations based on similar models, such as the CRN_TP model and Grandi et al. model (47), had been conducted to explain model dependence of results by reproducing Pitx2-induced AP shortening and triggered activity (18). Simulations with an alternative human atrial cell model should not alter the 

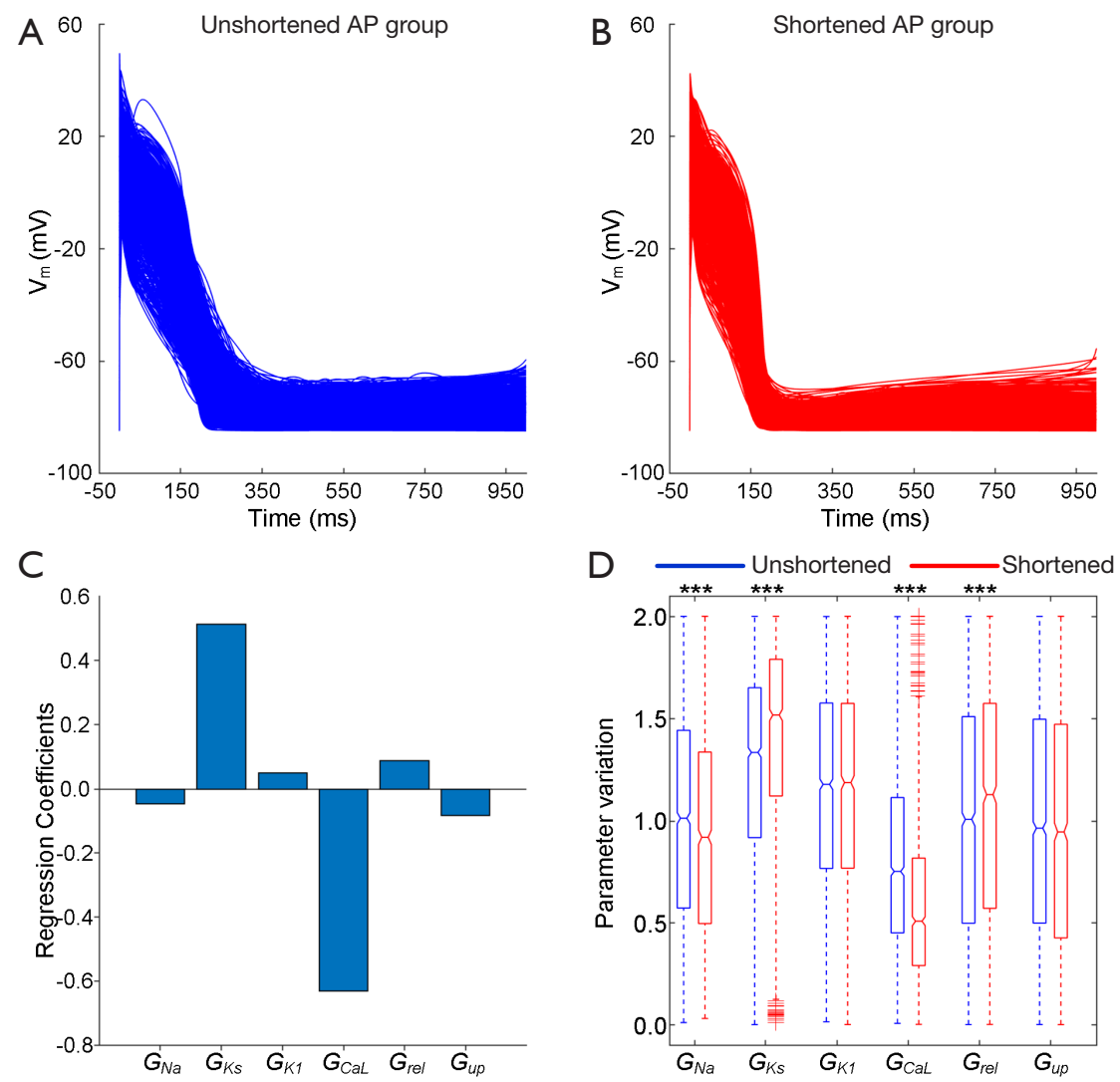

Figure 7 Action potential shortening in atrial fibrillation and underlying ionic parameters. (A,B) Representative traces of action potential (AP) models in the shortened versus unshortened groups. (C) Coefficients of the multinomial logistic regression model describing the probability of AP shortening in the models of our population. (D) Statistical analysis on the values of the perturbations to parameters modulated by Pitx2 in these two sub-groups of AP models. Each boxplot represents the range covered by the ionic conductances: the edges of the box are the $1^{\text {st }}$ and $3^{\text {rd }}$ quartiles, the whiskers extend to the most extreme datapoints, the estimated median physiological value is the central horizontal line and the notch around the median is the $5 \%$ significance level (Mann-Whitney $\mathrm{U}$ test: ${ }^{* * *}, \mathrm{P}<0.001$ ).

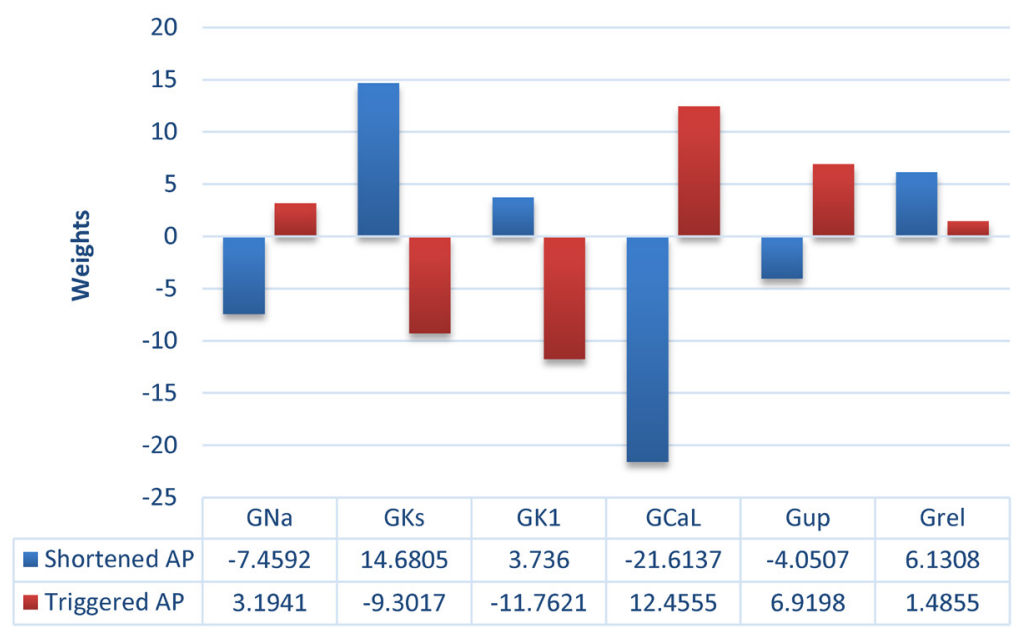

Figure 8 Weights of remodelled parameters (including $G_{\mathrm{Na}}, G_{\mathrm{Ks}}, G_{\mathrm{K} 1}, G_{\mathrm{CaL}}, \mathrm{G}_{\mathrm{Jrel}}$ and $\mathrm{G}_{\mathrm{up}}$ ) for classifying shortened and triggered AP three categories in AF models. 
Table 3 Weights of remodelled parameters (including $G_{\mathrm{Na}}, G_{\mathrm{Ks}}, G_{\mathrm{K} 1}, G_{\mathrm{CaL}}, G_{\mathrm{Jrel}}$ and $G_{\mathrm{up}}$ ) for classifying unshortened, shortened and triggered $A P$ three categories in AF models

\begin{tabular}{|c|c|c|c|c|c|c|}
\hline AP type & $\mathrm{G}_{\mathrm{Na}}$ & $\mathrm{G}_{\mathrm{Ks}}$ & $\mathrm{G}_{\mathrm{K} 1}$ & $\mathrm{G}_{\mathrm{CaL}}$ & $\mathrm{G}_{\text {up }}$ & $\mathrm{G}_{\mathrm{rel}}$ \\
\hline Shortened AP & -7.4592 & 14.6805 & 3.736 & -21.6137 & -4.0507 & 6.1308 \\
\hline Triggered AP & 3.1941 & -9.3017 & -11.7621 & 12.4555 & 6.9198 & 1.4855 \\
\hline
\end{tabular}

main findings of this study, although the inclusion of the intersubject variability approximates in silico experiments to more realistic clinical scenarios.

AP biomarkers from experimental recordings allowed us to select ionic current combinations that result in APs covering the observed experimental range. As in previous studies $(23,29)$, variability in ionic parameters reproduces AP variability in agreement with the biomarkers obtained from the recorded preparations. In order to account for Pixt2-induced triggered activity, we included restrictions in our population reproducing the observed phenomenon. To the best of our knowledge, this is the first time that AP morphology and APD relative to basal AP has been used to select the most physiologically relevant $\mathrm{AF}$ models from a wider population, therefore allowing a more realistic response of the models such as AP shortening and triggered activity observed during Pitx2-induced AF.

\section{Ionic mechanisms underlying Pitx2-induced AF}

Research studies on AF have demonstrated that AP shortening contributes to Pitx2-induced AF. An experimental study demonstrated AF increases Pitx2 expression in isolated human atrial myocytes and suggested Pitx 2 could contribute to the $I_{K s}$ increase and $I_{C a L}$ reduction observed in humans (3). Our modelling study further indicated the Pitx2-induced changes in $I_{K s}$ and $I_{C a L}$ led to APD shortening, facilitating sustained re-entry in $3 \mathrm{D}$ anatomical atrial geometry (17). However, studies on Pitx2-induced remodelling have not investigated the relative importance of each current in APD shortening. Our simulations in the AF population of models show AP shortening is primarily determined by $I_{C a L}$ and secondarily by $I_{K s}$. Furthermore, APD shortening is positively correlated with $I_{K s}$ and negatively correlated with $I_{C a L}$. This is consistent with findings from previous studies that upregulation of $I_{K s}(49)$ and downregulation of $I_{C a L}$ (50) due to Pitx2-induced remodelling critically contribute to the abbreviation of APD (3).
In addition to APD shortening (9), triggered activity (including DADs and spontaneous depolarizations) has been linked to Pitx2-induced remodelling (51). Experimental studies have demonstrated that Pitx2 was expressed in the left atrium and pulmonary veins (51), and reducing Pitx2 expression led to atrial remodelling linked to arrhythmogenesis (2). Some studies demonstrated that triggered activity is associated with the down-regulation of $I_{K 1}$ due to impaired Pitx2, resulting in a significantly depolarized resting membrane potential and thereby spontaneous depolarizations $(2,4)$. However, some studies indicated that multiple calcium-handling genes were regulated by Pitx2 $(3,8)$ and suggested that calcium handling abnormalities arising from Pitx2 insufficiency may contribute to triggered activity (18). Our simulations in the $\mathrm{AF}$ population of models show $\mathrm{DADs}$ can be induced by increased SR calcium load due to enhanced SERCA function. This is consistent with findings from these studies $(10,31)$ that the gain of SERCA function arising from the Pitx2 mutation contributed to calcium-mediated cellular triggered activity in AF. In addition to DADs, spontaneous depolarizations were obtained in the AF population of models. Our results showed that the inducibility of spontaneous depolarizations was positively and negatively correlated with $\mathcal{f}_{u p}$ and $I_{K 1}$, respectively. Although the pro-arrhythmic effect of downregulation of $I_{K 1}$ has been observed in simulations (20,52) and PV myocytes (53), the role of enhanced SERCA function due to Pitx2 insufficiency in the development of spontaneous depolarizations was not fully explored. Our results demonstrated that spontaneous depolarizations are primarily favored by low $I_{K 1}$, and secondarily by high $\mathcal{F}_{u p}$. These findings support this notion that spontaneous oscillations of membrane potential were indeed achieved upon a large increase in the SERCA pump activity (54). In addition, these observations for Pitx2induced remodeling, which includes upregulated $\mathcal{f}_{u p}$ and downregulated $I_{K 1}(2,4,7,8,10)$, are qualitatively similar to those observed here for the Pitx2 insufficiency. Therefore, the findings of the present study add to the growing weight 
to evidence implicating Pitx2-induced changes in $\mathcal{F}_{u p}$ and $I_{K I}$ in facilitating the triggered activity.

\section{The correlation of Pitx2 levels with different AF models}

Previous studies have shown that changes in Pitx2 levels lead to electrical remodelling linked to arrhythmogenesis (2) and that Pitx2 regulates membrane effector genes associated with the electrical remodelling in a dose-dependent manner $(3,7,8)$. Therefore, based on the correlation of the Pitx2 levels with remodelled parameters observed in previous studies $(3,4,7,8,41)$ and the correlation of remodelled parameters with changes in APs in the present study, the correlation of the Pitx2 levels with different AF models can be built. In the previous studies, Pitx 2 deficiency is associated with the increase in $G_{N a}, G_{u p}(8)$ and $G_{C a L}(41)$ and the reduction in $G_{K 1}(4)$. Interestingly, our simulated results indicated that the incidence of triggered $\mathrm{AP}$ was negatively correlated with $G_{K s}$ and $G_{K I}$ and was positively correlated with $G_{N a}, G_{C a L}$ and $G_{u p}$. Therefore, the downregulation of Pitx2 may increase the incidence of triggered AP. In addition, the study of Pérez-Hernández et al. (3) showed that Pitx 2 overexpression is associated with upregulated $G_{K s}$ and downregulated $G_{C a L}$. And our simulated results showed that the incidence of shortened AP was positively correlated with $G_{K s}$ and $G_{K 1}$ and was negatively correlated with $G_{N a}, G_{C a L}$ and $G_{u p}$. Therefore, the upregulation of Pitx2 may favor to AP shortening. Thus, the Pitx2 level may be positively correlated with the occurrence of AP shortening, but negatively correlated with the incidence of triggered AP.

\section{Pitx2 and AF-relevance to previous studies}

Gain-of-function/loss-of-function of Pitx2 has been found to be associated with $\mathrm{AF}$ in humans $(2-4,9,10,19,21)$. A number of previous studies have shown expression levels of Pitx2 are reduced in the left atrium in humans $(2,4,55)$ and transgenic mice $(2,8,9)$, or with loss-of-function mutations $(56,57)$. However, Pitx 2 expression has been found to be increased in the left atrium (58) and in right atrial myocytes (3), from $\mathrm{AF}$ patients, or with the gain-of-function mutation p.Met207Val (59). The present study is consistent with this notion, indicating clearly a marked function impact of gain-of-function/loss-of-function of PITX2 on atrial cell electrophysiology that would promote susceptibility to AF. In previous simulated studies, we have shown that a shorten $\mathrm{APD}_{90}$ arising from the Pitx2 overexpression (17) or the gain-of-function mutation p.Met207Val (19) increased atrial susceptibility to arrhythmias, and that abnormalities in intracellular calcium handling due to the Pitx2 downregulation $(18,20)$ or the loss-of-function mutation rs13143308T (31) increased the inducibility of triggered activity which can be suppressed by drugs such as dantrolene and flecainide. Our data are consistent with this. They indicate that Pitx2-induced electrical remodelling not only contributes to the $\mathrm{APD}_{90}$ shortening in spatial vulnerability to arrhythmia, but also increases the susceptibility of the human atrial cell to the genesis of triggered activity. Therefore, our study substantiates a causative link between Pitx2-induced electrical remodelling and AF.

Accumulating evidence places particular emphasis on atrial arrhythmogenicity due to intersubject variability in Pitx2 levels (9), in producing the electrical remodelling in a dose-dependent manner $(3,7)$. The present study is both consistent with and extends this notion, indicating a relationship between the PITX2 alteration and arrhythmia onset. The general incidence of $\mathrm{AF}$ increases with age. The previous study of Scridon et al. have shown that PITX2 expressions showed a progressive, age-dependent change and were negatively correlated with both age and heart weight in hypertensive rats (5). It indicated a possible temporal relationship between the PITX2 alteration and arrhythmia onset, suggesting PITX2 alteration is an agedependent process that starts before the occurrence of arrhythmias. And Lozano-Velasco et al. identified a complex regulatory network orchestrated by PITX2, demonstrating a dose-dependent relation between PITX2 expression and the expression of AF susceptibility genes (7). Importantly, human right atrial myocytes from $\mathrm{AF}$ patients have shown PITX2 expression increases and this increase correlates with the $I_{K s}$ increase and $I_{C a L}$ decrease that characterizes AFinduced electrical remodeling, demonstrating the substrate for arrhythmogenesis in these AF patients is dependent upon electrical remodeling modulated by the PITX2 expression in a dose-dependent manner (3). Our data are consistent with this. They indicated that the incidence of shortened AP was correlated with the extent of electrical remodelling due to Pitx2 upregulation and the incidence of triggered activity was correlated with the extent of electrical remodelling due to Pitx2 downregulation. Therefore, the correlation of the Pitx2 levels with arrhythmia onset with different mechanisms was built.

\section{Study limitations}

Several limitations special to the present study are 
summarized. Fristly, the populations of human atrial models developed in the present study are based on a large number of experimental recordings obtained from the right atrial appendage. Cardiomyocytes from other atrial locations may exhibit spatial heterogeneities in AP properties reported in humans (60-62). These heterogeneities were not directly addressed in the present study, but our population approach does offer robustness against variability resulting from this. Furthermore, the inter-cellular coupling may affect APs as compared to isolated cells. The effects of tissue coupling could be investigated in further studies, aiming at translating the conclusions obtained from cellular to tissue model populations. Secondly, because of the lack of relevant experimental data, the potential impact of Pitx2 was evaluated under the following assumption: The Pitx2induced functional changes in the proteins would be proportional to their mRNAs changes. Our study inherits many of the difficulties in extrapolating from mRNA expression data to functional membrane ion channels. In addition, we examined the effects of variability in a subset of conductances associated with Pitx2 in the present study. However, it is possible that other electrophysiological properties, such as ionic concentrations and additional ionic currents, may play a role in AP variability. These additional important factors could be the focus of future investigations. Although these potential limitations are important in the present study, they may not influence our conclusions drawn from simulated results on the mechanisms by which Pitx2-induced remodelling can increase the risk of AF.

\section{Conclusions}

Experimentally calibrated populations of models are presented as a useful tool for understanding the ionic mechanisms underlying Pitx2-induced AF. This study showed that the populations of models reproduce the variability in human atrial AP properties measured in samples obtained from patients and main ionic mechanisms modulating intersubject variability in the different phases of the $\mathrm{AP}$ are very similar in $\mathrm{SR}$ and $\mathrm{AF}$ populations. The $\mathrm{AF}$ population of models predicts AP shortening and triggered activity in Pitx2-induced remodelling conditions observed in experiments. By using this computational framework, our results suggest that the incidence of shortened AP was positively correlated with $I_{K s}$ and $I_{K l}$ and was negatively correlated with $I_{N a}, I_{C a L}$ and SERCA, whereas the incidence of triggered AP was negatively correlated with $I_{K s}$ and $I_{K 1}$ and was positively correlated with $I_{N a}, I_{C a L}$ and SERCA. Based on the correlation of Pitx2-induced remodelling with different $\mathrm{AF}$ models and the correlation of Pitx2 levels with the electrical remodelling in previous studies, we can conclude that the Pitx2 level may be positively correlated with the occurrence of AP shortening, but negatively correlated with the incidence of triggered AP. Therefore, the outcomes of this study may lead to more targeted patient-specific treatment for Pitx2-induced AF in the near future.

\section{Acknowledgments}

We would like to thank Andy Lo and Jichao Zhao for their help in polishing the language of our paper.

Funding: This work was supported by the National Natural Science Foundation of China (No. 61901192) and the National Key Research and Development Project (No. 2019YFC0120100 and 2019YFC0121907).

\section{Footnote}

Reporting Checklist: The authors have completed the MDAR reporting checklist. Available at http://dx.doi.org/10.21037/ atm-20-5660

Data Sharing Statement: Available at http://dx.doi. org/10.21037/atm-20-5660

Peer Review File: Available at http://dx.doi.org/10.21037/ atm-20-5660

Conflicts of Interest: All authors have completed the ICMJE uniform disclosure form (available at http://dx.doi. org/10.21037/atm-20-5660). The authors have no conflicts of interest to declare.

Ethical Statement: The authors are accountable for all aspects of the work in ensuring that questions related to the accuracy or integrity of any part of the work are appropriately investigated and resolved.

Open Access Statement: This is an Open Access article distributed in accordance with the Creative Commons Attribution-NonCommercial-NoDerivs 4.0 International License (CC BY-NC-ND 4.0), which permits the noncommercial replication and distribution of the article with the strict proviso that no changes or edits are made and the original work is properly cited (including links to both the formal publication through the relevant DOI and the license). 
See: https://creativecommons.org/licenses/by-nc-nd/4.0/.

\section{References}

1. Gudbjartsson DF, Arnar DO, Helgadottir A, et al. Variants conferring risk of atrial fibrillation on chromosome 4q25. Nature 2007;448:353-7.

2. Chinchilla A, Daimi H, Lozano-Velasco E, et al. PITX2 insufficiency leads to atrial electrical and structural remodeling linked to arrhythmogenesis. Circ Cardiovasc Genet 2011;4:269-79.

3. Pérez-Hernández $M$, Matamoros $M$, Barana A, et al. Pitx2c increases in atrial myocytes from chronic atrial fibrillation patients enhancing I Ks and decreasing I Ca, L. Cardiovasc Res 2016;109:431-41.

4. Syeda F, Holmes AP, Ting YY, et al. PITX2 modulates atrial membrane potential and the antiarrhythmic effects of sodium-channel blockers. J Am Coll Cardiol 2016;68:1881-94.

5. Scridon A, Fouilloux-Meugnier E, Loizon E, et al. Longstanding arterial hypertension is associated with Pitx2 down-regulation in a rat model of spontaneous atrial tachyarrhythmias. Europace 2015;17:160-5.

6. Nattel S, Frelin Y, Gaborit N, et al. Ion-channel mRNAexpression profiling: Insights into cardiac remodeling and arrhythmic substrates. J Mol Cell Cardiol 2010;48:96-105.

7. Lozano-Velasco E, Hernandez-Torres F, Daimi H, et al. Pitx2 impairs calcium handling in a dose-dependent manner by modulating Wnt signalling. Cardiovasc Res 2016;109:55-66.

8. Nadadur RD, Broman MT, Boukens B, et al. Pitx2 modulates a $\mathrm{Tbx} 5$-dependent gene regulatory network to maintain atrial rhythm. Sci Transl Med 2016;8:354ra115.

9. Kirchhof P, Kahr PC, Kaese S, et al. PITX2c is expressed in the adult left atrium, and reducing Pitx2c expression promotes atrial fibrillation inducibility and complex changes in gene expression. Circ Cardiovasc Genet 2011;4:123-33.

10. Herraiz-Martínez A, Llach A, Tarifa C, et al. The 4q25 variant rs $13143308 \mathrm{~T}$ links risk of atrial fibrillation to defective calcium homoeostasis. Cardiovasc Res 2019;115:578-89.

11. Bray MS, Shaw CA, Moore MW, et al. Disruption of the circadian clock within the cardiomyocyte influences myocardial contractile function, metabolism, and gene expression. Am J Physiol Heart Circ Physiol 2008;294:H1036-47.

12. Jeyaraj D, Haldar SM, Wan X, et al. Circadian rhythms govern cardiac repolarization and arrhythmogenesis. Nature 2012;483:96-9.

13. Gaborit N, Varro A, Le Bouter S, et al. Gender-related differences in ion-channel and transporter subunit expression in non-diseased human hearts. J Mol Cell Cardiol 2010;49:639-46.

14. Ambrosi CM, Yamada KA, Nerbonne JM, et al. Gender differences in electrophysiological gene expression in failing and non-failing human hearts. PLoS One 2013;8:e54635.

15. James AF, Choisy SC, Hancox JC. Recent advances in understanding sex differences in cardiac repolarization. Prog Biophys Mol Biol 2007;94:265-319.

16. Soltysinska E, Olesen SP, Christ T, et al. Transmural expression of ion channels and transporters in human nondiseased and end-stage failing hearts. Pflugers Archiv 2009;459:11-23.

17. Bai J, Lu Y, Lo A, et al. PITX2 upregulation increases the risk of chronic atrial fibrillation in a dose-dependent manner by modulating IKs and ICaL-insights from human atrial modelling. Ann Transl Med 2020;8:191.

18. Bai J, Lo A, Gladding PA, et al. In silico investigation of the mechanisms underlying atrial fibrillation due to impaired Pitx2. PLoS Comput Biol 2020;16:e1007678.

19. Bai J, Lu Y, Lo AC, et al. Proarrhythmia in the p. Met207Val PITX2c-linked familial atrial fibrillationinsights from modelling. Front Physiol 2019;10:1314.

20. Bai J, Gladding PA, Stiles MK, et al. Ionic and cellular mechanisms underlying TBX5/PITX2 insufficiencyinduced atrial fibrillation: Insights from mathematical models of human atrial cells. Sci Rep 2018;8:15642.

21. Tao Y, Zhang M, Li L, et al. Pitx2, an atrial fibrillation predisposition gene, directly regulates ion transport and intercalated disc genes. Circ Cardiovasc Genet 2014;7:23-32.

22. Britton OJ, Bueno-Orovio A, Van Ammel K, et al. Experimentally calibrated population of models predicts and explains intersubject variability in cardiac cellular electrophysiology. Proc Natl Acad Sci USA 2013;110:E2098-E2105.

23. Sánchez C, Bueno-Orovio A, Wettwer E, et al. Intersubject variability in human atrial action potential in sinus rhythm versus chronic atrial fibrillation. PloS One 2014;9:e105897.

24. Lawson BA, Drovandi CC, Cusimano N, et al. Unlocking data sets by calibrating populations of models to data density: A study in atrial electrophysiology. Sci Adv 2018;4:e1701676. 


\section{Page 16 of 17}

25. Muszkiewicz A, Liu X, Bueno-Orovio A, et al. From ionic to cellular variability in human atrial myocytes: an integrative computational and experimental study. Am J Physiol Heart Circ Physiol 2018;314:H895-H916.

26. Coveney S, Clayton RH. Fitting two human atrial cell models to experimental data using Bayesian history matching. Prog Biophys Mol Biol 2018;139:43-58.

27. Vagos MR, Arevalo H, De Oliveira BL, et al. A computational framework for testing arrhythmia marker sensitivities to model parameters in functionally calibrated populations of atrial cells. Chaos 2017;27:093941.

28. Coveney S, Clayton RH. Sensitivity and uncertainty analysis of two human atrial cardiac cell models using Gaussian process emulators. Front Physiol 2020;11:364.

29. Liberos A, Bueno-Orovio A, Rodrigo M, et al. Balance between sodium and calcium currents underlying chronic atrial fibrillation termination: An in silico intersubject variability study. Heart Rhythm 2016;13:2358-65.

30. Bai J, Lu Y, Lo AC, et al. PITX2 overexpression leads to atrial electrical remodeling linked to atrial fibrillation. Proceedings of the 2019 Computing in Cardiology (CinC).

31. Bai J, Zhu Y, Lu Y, et al. In silico assessment of genetic variation in PITX2 reveals the molecular mechanisms of calcium-mediated cellular triggered activity in atrial fibrillation. Proceedings of the 2020 42nd Annual International Conference of the IEEE Engineering in Medicine and Biology Society (EMBC).

32. Dobrev D, Wettwer E, Himmel HM, et al. G-Protein beta(3)-subunit $825 \mathrm{~T}$ allele is associated with enhanced human atrial inward rectifier potassium currents. Circulation 2000;102:692-7.

33. Wettwer E, Christ T, Endig S, et al. The new antiarrhythmic drug vernakalant: ex vivo study of human atrial tissue from sinus rhythm and chronic atrial fibrillation. Cardiovasc Res 2013;98:145-54.

34. Wettwer E, Hála O, Christ T, et al. Role of IKur in controlling action potential shape and contractility in the human atrium: influence of chronic atrial fibrillation. Circulation 2004;110:2299-306.

35. Morrison TM, Hariharan P, Funkhouser CM, et al. Assessing computational model credibility using a riskbased framework: application to hemolysis in centrifugal blood pumps. ASAIO J 2019;65:349-60.

36. Passini E, Mincholé A, Coppini R, et al. Mechanisms of pro-arrhythmic abnormalities in ventricular repolarisation and anti-arrhythmic therapies in human hypertrophic cardiomyopathy. J Mol Cell Cardiol 2016;96:72-81.

37. Trovato C, Passini E, Nagy N, et al. Human Purkinje
Zhu et al. Mechanisms underlying Pitx2-induced atrial fibrillation

in silico model enables mechanistic investigations into automaticity and pro-arrhythmic abnormalities. J Mol Cell Cardiol 2020;142:24-38.

38. Marino S, Hogue IB, Ray CJ, et al. A methodology for performing global uncertainty and sensitivity analysis in systems biology. J Theor Biol 2008;254:178-196.

39. Morotti S, Grandi E. Logistic regression analysis of populations of electrophysiological models to assess proarrythmic risk. MethodsX 2016;4:25-34.

40. Dreiseitl S, Ohno-Machado L. Logistic regression and artificial neural network classification models: a methodology review. J Biomed Inform 2002;35:352-9.

41. Dai W, Laforest B, Tyan L, et al. A calcium transport mechanism for atrial fibrillation in Tbx5-mutant mice. eLife 2019;8:e41814.

42. Lugo CA, Cantalapiedra IR, Peñaranda A, et al. Are SR Ca content fluctuations or SR refractoriness the key to atrial cardiac alternans?: insights from a human atrial model. Am J Physiol Heart Circ Physiol 2014;306:H1540-H1552.

43. Maleckar MM, Greenstein JL, Giles WR, et al. K+ current changes account for the rate dependence of the action potential in the human atrial myocyte. Am J Physiol Heart Circ Physiol 2009;297:H1398-H1410.

44. Nygren A, Fiset C, Firek L, et al. Mathematical Model of an Adult Human Atrial Cell. Circ Res 1998;82:63-81.

45. Koivumäki J T, Korhonen T, Tavi P. Impact of Sarcoplasmic Reticulum Calcium Release on Calcium Dynamics and Action Potential Morphology in Human Atrial Myocytes: A Computational Study. PLoS Comput Biol 2011;7:e1001067.

46. Courtemanche M, Ramirez RJ, Nattel S. Ionic mechanisms underlying human atrial action potential properties: insights from a mathematical model. Am J Physiol 1998;275:H301-H321.

47. Grandi E, Pandit SV, Voigt N, et al. Human Atrial Action Potential and Ca2+ Model. Circ Res 2011;109:1055-66.

48. Voigt N, Heijman J, Wang Q, et al. Cellular and Molecular Mechanisms of Atrial Arrhythmogenesis in Patients With Paroxysmal Atrial Fibrillation. Circulation 2014;129:145-56.

49. González de la Fuente M, Barana A, Gómez R, et al. Chronic atrial fibrillation up-regulates $\beta 1$-Adrenoceptors affecting repolarizing currents and action potential duration. Cardiovasc Res 2013;97:379-88.

50. Van Wagoner DR, Pond AL, Lamorgese M, et al. Atrial L-Type Ca2+ > Currents and Human Atrial Fibrillation. Circ Res 1999;85:428-36.

51. Wang J, Klysik E, Sood S, et al. Pitx2 prevents 
susceptibility to atrial arrhythmias by inhibiting leftsided pacemaker specification. Proc Natl Acad Sci USA 2010;107:9753-8.

52. Kurata Y, Hisatome I, Matsuda H, et al. Dynamical Mechanisms of Pacemaker Generation in IK1Downregulated Human Ventricular Myocytes: Insights from Bifurcation Analyses of a Mathematical Model. Biophys J 2005;89:2865-87.

53. Chen YJ, Chen SA, Chen YC, et al. Effects of rapid atrial pacing on the arrhythmogenic activity of single cardiomyocytes from pulmonary veins. Circulation 2001;104:2849-54.

54. Fabbri A, Fantini M, Wilders R, et al. Computational analysis of the human sinus node action potential: model development and effects of mutations. J Physiol 2017;595:2365-96.

55. Gore-Panter SR, Hsu J, Barnard J, et al. PANCR, the PITX2 Adjacent Noncoding RNA, Is Expressed in Human Left Atria and Regulates PITX2c Expression. Circ Arrhythm Electrophysiol 2016;9:e003197.

56. Qiu XB, Xu YJ, Li RG, et al. PITX2C loss-of-function mutations responsible for idiopathic atrial fibrillation.

Cite this article as: Zhu Y, Bai J, Lo A, Lu Y, Zhao J. Mechanisms underlying pro-arrhythmic abnormalities arising from Pitx2-induced electrical remodelling: an in silico intersubject variability study. Ann Transl Med 2021;9(2):106. doi: $10.21037 /$ atm-20-5660
Clinics (Sao Paulo) 2014;69:15-22.

57. Wang J, Xin YF, Xu WJ, et al. Prevalence and spectrum of PITX2c mutations associated with congenital heart disease. DNA Cell Biol 2013;32:708-16.

58. Gore-Panter SR, Hsu J, Hanna P, et al. Atrial Fibrillation associated chromosome $4 \mathrm{q} 25$ variants are not associated with PITX2c expression in human adult left atrial appendages. PLoS One 2014;9:e86245.

59. Mechakra A, Footz T, Walter M, et al. A Novel PITX2c Gain-of-Function Mutation, p.Met207Val, in Patients With Familial Atrial Fibrillation. Am J Cardiol 2019;123:787-93.

60. Feng J, Yue L, Wang Z, et al. Ionic Mechanisms of Regional Action Potential Heterogeneity in the Canine Right Atrium. Circ Res 1998;83:541-51.

61. Li D, Zhang L, Kneller J, et al. Potential Ionic Mechanism for Repolarization Differences Between Canine Right and Left Atrium. Circ Res 2001;88:1168-75.

62. Gong D, Zhang Y, Cai B, et al. Characterization and Comparison of $\mathrm{Na}+\mathrm{K}+$ and $\mathrm{Ca} 2+$ Currents Between Myocytes from Human Atrial Right Appendage and Atrial Septum. Cell Physiol Biochem 2008;21:385-94. 